\title{
Design and Implementation of 64-Bit RISC Processor for Industry Automation
}

\author{
P. Devi Pradeep ${ }^{1}$ and D.Srinivasa $\mathrm{Rao}^{2}$ \\ ${ }^{1,2}$ Assistant Professor, Department of ECE, GMR Institute of Technology, RAJAM, \\ A.P, India \\ devipradeep.p@gmrit.org,srinivasarao.d@gmrit.org
}

\begin{abstract}
In the era of industrialization, technological revolution reduced the intervention of humans to assist machinery. The privatization slogan has increased the competition among industries which leads to origin of new products/designs for automation. The paper presents, one such, design and implementation of 64-bit RISC processor on SOC for industry automation, mainly useful for packing of bottles in bottling plant. The design includes processor with BIST features; it is a mechanism that allows a machine to test itself. Here RISC processor is used to decrease the number of instructions and they execute the instruction at faster rate. Later the design is integrated on System on Chip (SoC) to obtain a single chip which reduces the overall power consumption. The design and synthesis is done by verilog and verified in cadence IUS and RTL compiler. The physical design is done by SoC encounter 11.10 version.
\end{abstract}

Keywords: RISC, SoC, BIST

\section{Introduction}

The technology can take beverage industry beyond competition and market entails opportunities that can be transformed into success. High degree of flexibility is its prior need. Also industries face many other challenges. The pressure to continually increase production volumes has stressed older systems and has increased maintenance requirements. For manufacturers, this creates two problems: higher costs and increased downtime. Production managers are being challenged to reduce cost, wastage and downtime. New technologies are required that will reduce water usage, increase energy efficiency and minimize downtime in high-speed beverage production environments. Increasing competitive pressures, ever more stringent legal regulations, rising costs of commodities and energy and consumers whose preferences are subject to rapid change - beverage companies today are forced to increase their flexibility and operate with maximum efficiency at the ame time. The key to this problem is an integrated process approach. After all, if all processes are perfectly coordinated with each other and reliable communications have been established between all parts of the manufacturing plant, it is much easier to address the big challenges [1].

\section{System On Chip}

System on chip is an integrated circuit that integrates all components of computer or other electronic system into single chip. It may contain analog, digital and mixed signal functions all on a single chip substrate. A typical application is in the area of embedded systems.

A typical soc consists of 
1. Microcontroller and microprocessor and multiprocessors furthermore.

2. Memory blocks including RAM, ROM, and flash memory.

3. Timing controllers together with oscillators and phase locked loops.

4. External interfaces including industry standards such as USB, Fire Wire, Ethernet, USART, and SPI. In this paper soc is done by using FIRST ENCOUNTER 11.10 version.

\section{Proposed Architecture}

In this proposed architecture the clock generator generates the three clock cycles and gives that three clock cycles to control and decoder unit. Then that unit will set LdIr port to 1 which means that it is telling to the instruction register to load the data from the memory or internal registers. After loading the data that data is given to program counter and control and decoder unit. In our project the data will be 64-bit data. In that data first 6 LSB bits are for memory address, last four MSB bits are for OPCODE, the next 3 MSB bits are for operand source address and the next 3 MSB bits are for operand destination address. According to that addresses the control and decoder unit will load the operands from the memory or internal registers at the time of loading operand from the memory it will set the ports MemRd to 1 and MemWr to 0 which means that memory read operation is going on. The data will be loaded into MuxA. If we want to do any ALU operation we need two operands already one operand is loaded into the MuxA the other operand will also be loaded into the MuxB in the same way. At the time of selecting the internal registers the control and decoder unit will tell which register we have to select using the operand source address. Then the outputs (data) from two Multiplexers are loaded into the ALU unit where the execution of operation will be done. At that time the control and decoder unit will tell which operation we want to do using the opcode.

After doing the ALU operation that output can be stored in either internal registers or memory. If we want to store the data in the internal registers then the control and decoder unit will select the destination register using the operand destination address. And if we want to store the data in the memory then the control and decoder unit will set the MemWr tol and MemRd to 0 so that we can write the data into the memory. For selecting the address to where we want to store the data in the memory it will use the memory address in the opcode and if we want to use the different address location the program counter will increment the location so that we can store the data to that address location, this address location will be selected by using a 2:1 mux with a fetch select line under the control of control and decoder unit. While storing the output data from the alu unit into the internal registers the buffer will be in high impedance state which means that it will not allow the data to flow into the memory unit. And while storing the output data into the memory the buffer will be set to 1 so that the data will be loaded into memory. 


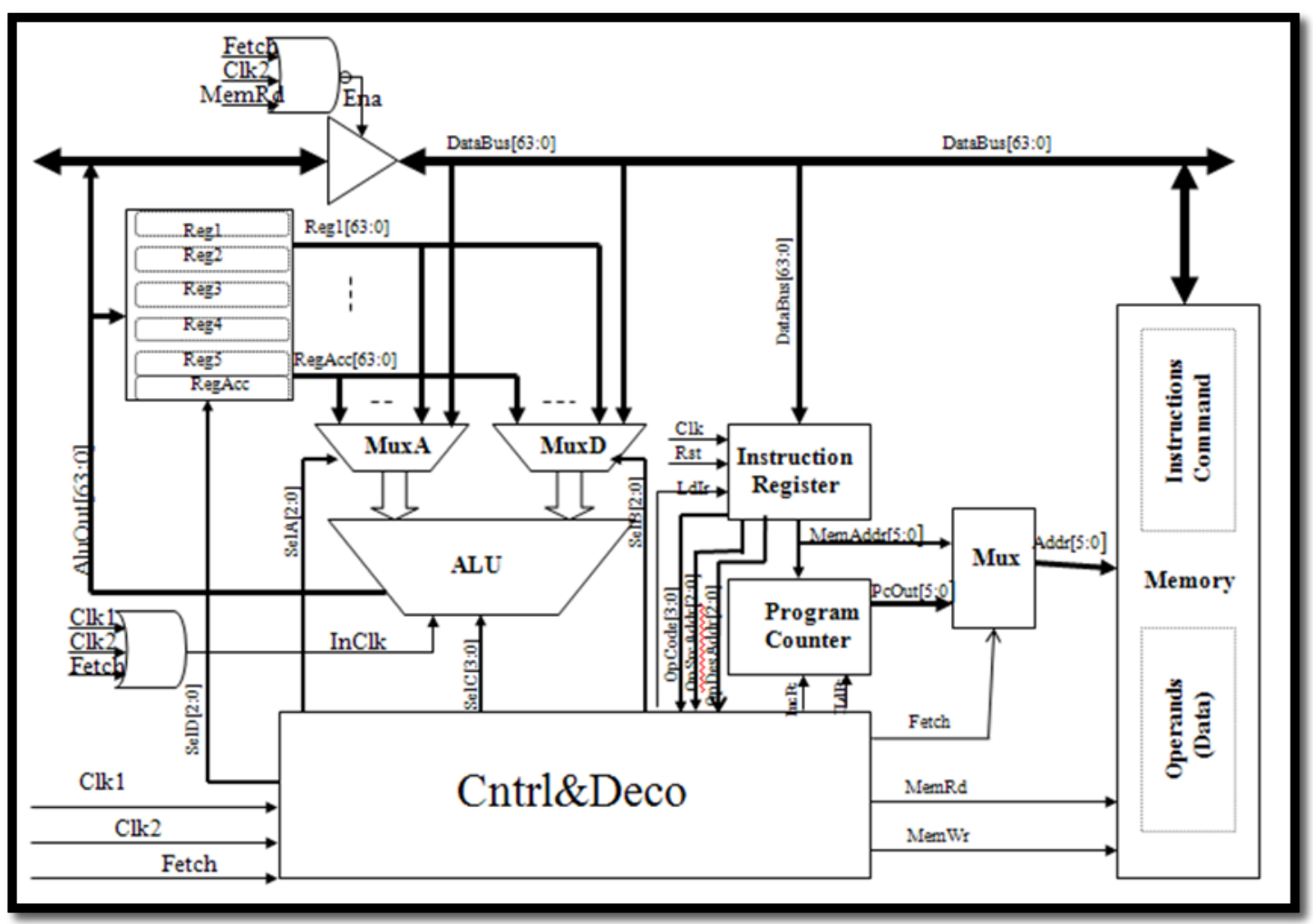

Figure 1. 64-bit RISC Architecture with Entire Design Flow

\section{Simulation Results:}

The proposed architecture is described in verilog. Design and testing of each individual module has been carried out. Figure 3 depicts the simulation results of alu addition and fig. 4 depicts the the power utilization report and delay report. Figure 5 depicts controller unit and Figure 6 depicts the SoC of the bottling plant.

\section{Application:}

Bottling plant: Bottles start satisfying from the exact side and boxes start to move from the absent side. Here four tracks of bottles are used concurrently therefore protective material is made of four bottles. When bottle reaches to the fourth arrangement, box moves to the first position. After that, bottle is dropped in the box and hence, box moves one position ahead. In this way, when box is at the fifth position, signal ' $1 b$ ' is set to ' 1 ' representing to lift the box. 


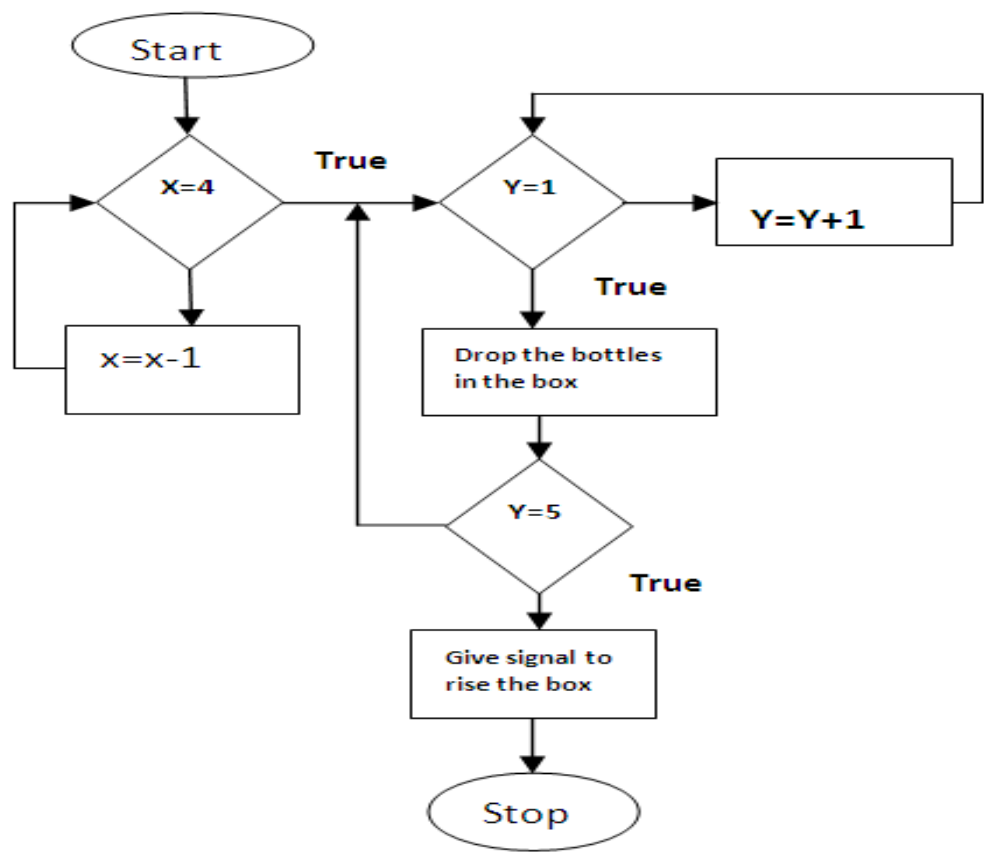

Figure 2. RISC Processor Flow for Bottling Plant

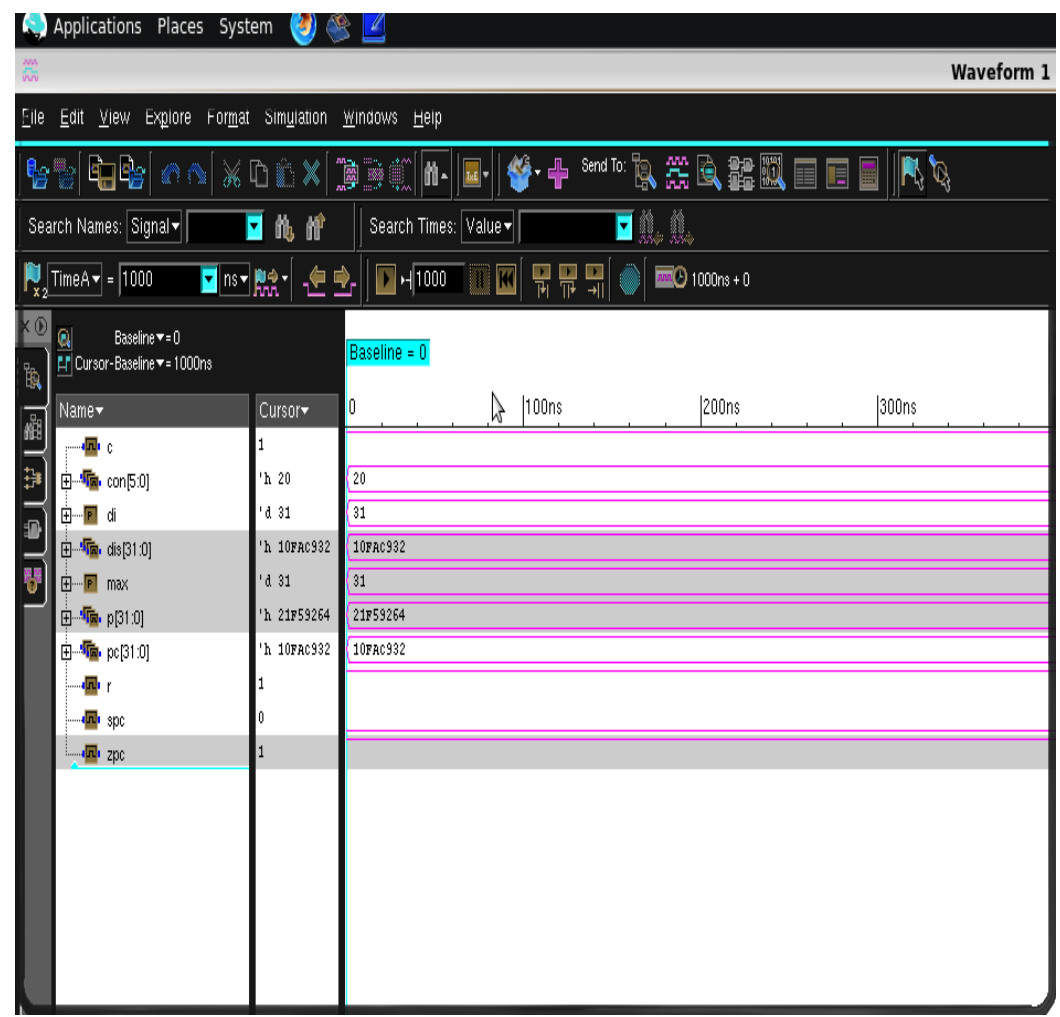

Figure 3. Simulation Results of RISC Processor 


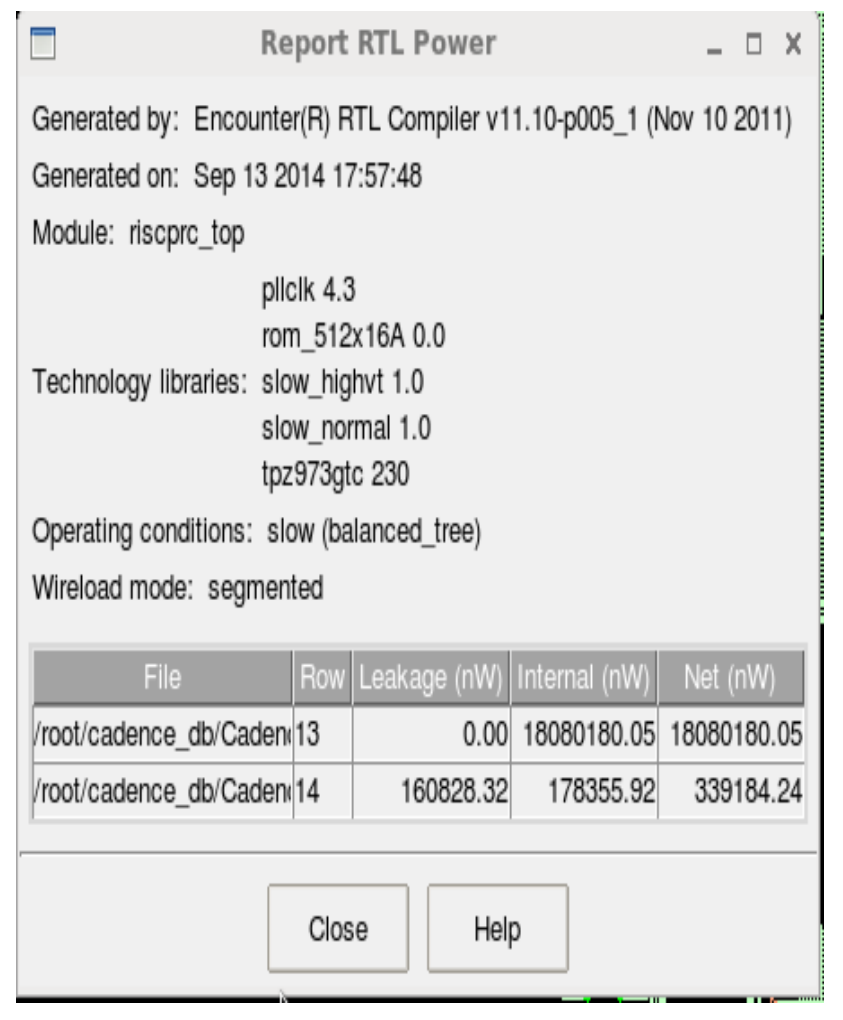

Figure 4: Power Utilization and Delay Report

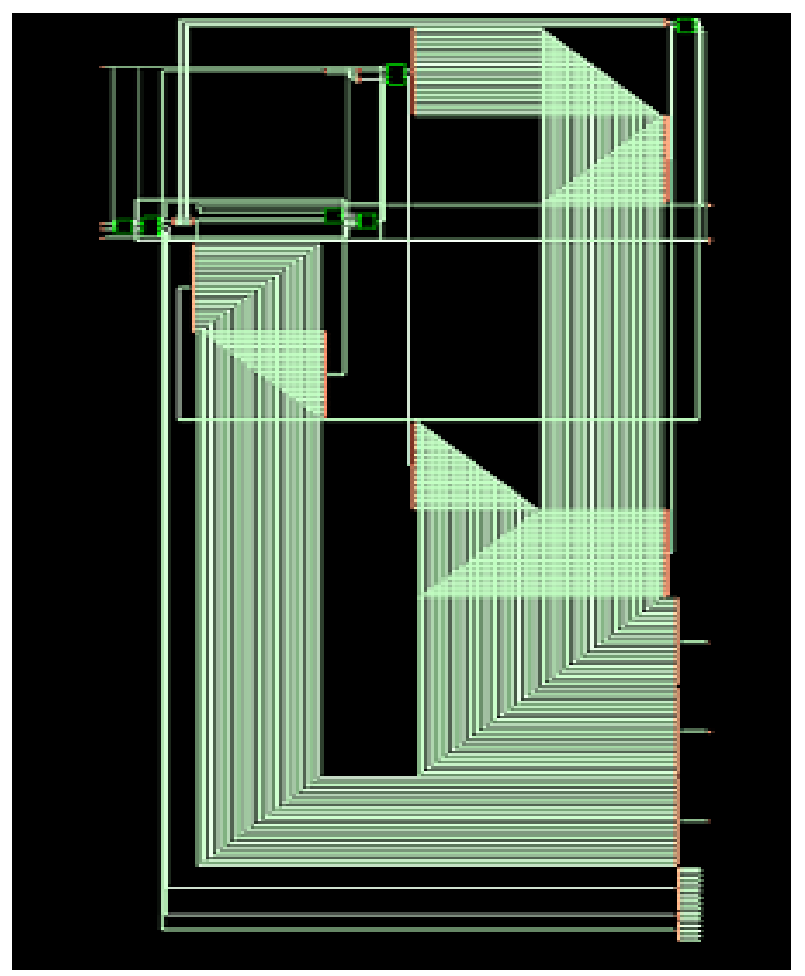

Figure 5. Program Counter 


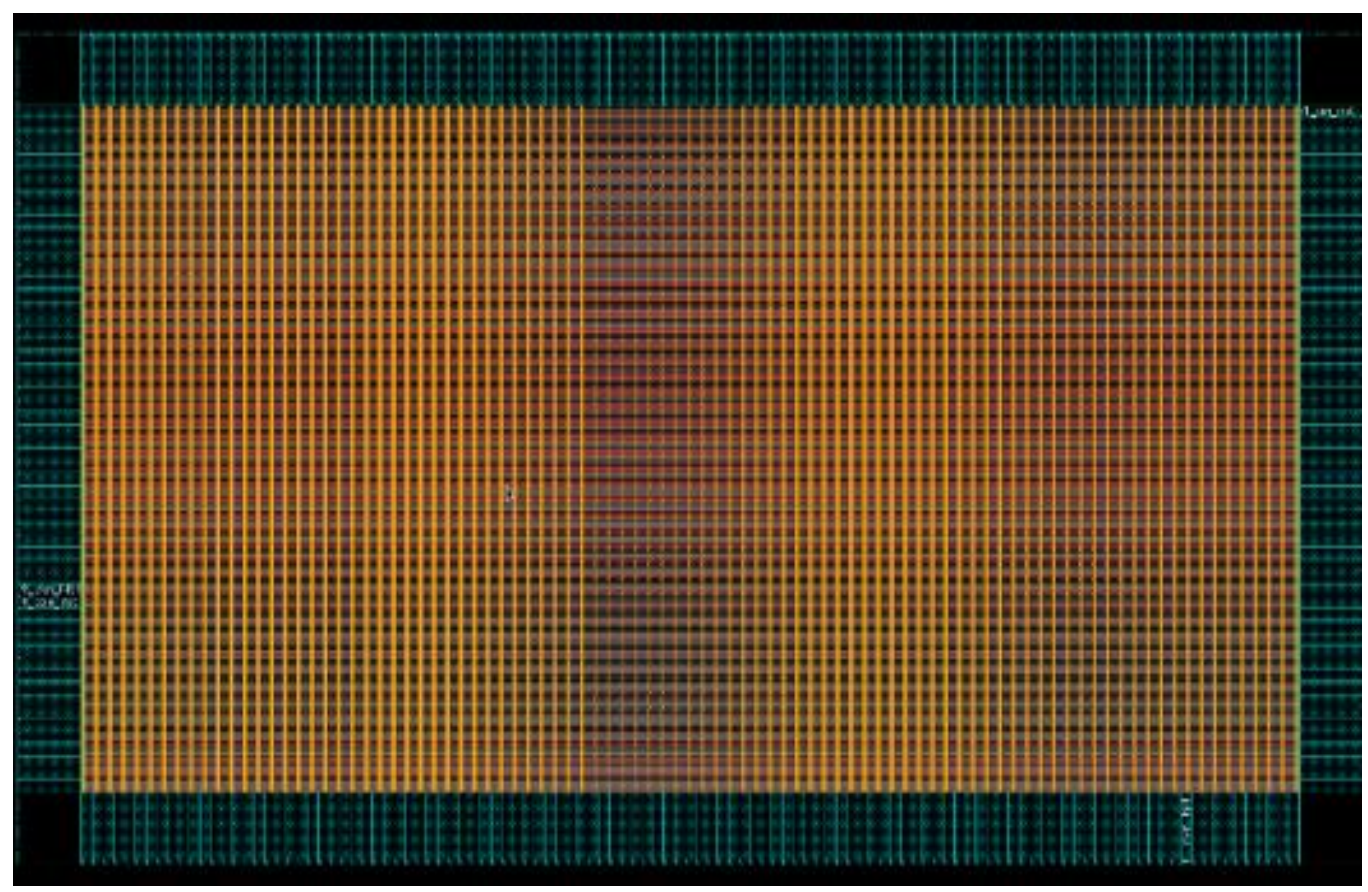

Figure 6. SOC of Bottling Plant

\section{Conclusion}

Various individual modules of Our project have been designed, verified functionally using Verilog HDL-simulator (Active HDL), synthesized by the RTL compiler with IUS(cadence) tool. This design of the 64-bit RISC Processor is capable of performing arithmetic and logical operations with the help of ALU block. The control and decoder unit controls all the modules. The Functional-simulation has been successfully carried out with designed processor is also capable of performing control instruction like JUMP, SKIP and HALT. The Functionalsimulation has been successfully carried out with the results matching with the expected ones. Within this the power consumed is $18080.18 \mathrm{uw}$.

\section{References}

[1] Rohit sharma, design and implementation of 64-bit RISC processor using VHDL, $11^{\text {th }}$ International conference on computer modeling and simulation, IEEE 2009.

[2] Samuel O. Aletan, "An Overview of RISC Architecture, Proc. Symp. Applied Computing, 1992, pp. 11-12.

[3] D. Bhandarkar and D. W. Clark, "Performance from Architecture: comparing a RISC and a CISC with similar Hardware Organization," Proc. 4th International Conference on Architectural Support for Programming Languages and Operating Systems, 1991, pp. 310-319.

[4] T. Marshall, "Worth the RISC," The BYTE, vol. 14, no. 2, 1989, pp. 245-249.

[5] R. N. Noyce and M. E. Hoff, "A History of Microprocessor Development at Intel," IEEE Micro, vol. 1, no. 1, 1981, pp.8-21.

[6] J.L. Hennessy, "VLSI Processor Architecture," IEEE Trans. Computers, vol. C-33, no. 12, Dec. 1984, pp. 1221-1246.

[7] Design and Implementation of a 64-bit RISC Processor using VHDL, 2009 IEEE. 


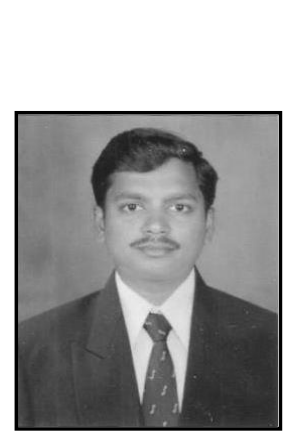

\section{Author}

P. Devi Pradeep received M.Tech degree from National Institute of Technology, Warangal, Andhra Pradesh, India. B.Tech degree in Electronics and Communication Engineering from Vitam College of engineering affiliated to JNTU Hyderabad, Andhra Pradesh, India. He is joined as Assistant Professor in the Department. Of Electronics and Communication Engineering at GMR Institute of Technology, Rajam, Srikakulam District, Andhra Pradesh, India in 2009. Prior to join in this Institute he worked as a Assistant Professor in the VITAM college of engineering. Having Total teaching experience is 9 years. His research interests are Video Processing, Analog and digital VLSI Design. He is a life member of ISTE Since 2009. 
International Journal of $u$ - and e- Service, Science and Technology Vol.8, No.1 (2015) 